Article

\title{
Durrmeyer-Type Generalization of Parametric Bernstein Operators
}

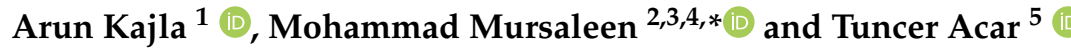 \\ 1 School of Basic Sciences, Faculty of Mathematics, Central University of Haryana, Haryana-123029, India; \\ arunkajla@cuh.ac.in \\ 2 Department of Medical Research, China Medical University Hospital, China Medical University, \\ Taichung 40402, Taiwan \\ 3 Department of Mathematics, Aligarh Muslim University, Aligarh 202002, India \\ 4 Department of Computer Science and Information Engineering, Asia University, Taichung 41354, Taiwan \\ 5 Department of Mathematics, Faculty of Science, Selcuk University, Selcuklu, Konya 42003, Turkey; \\ tuncer.acar@selcuk.edu.tr \\ * Correspondence: mursaleen.mm@amu.ac.in; Tel.: +91-941-149-1600
}

Received: 13 June 2020; Accepted: 6 July 2020; Published: 8 July 2020

check for updates

\begin{abstract}
In this paper, we present a Durrmeyer type generalization of parametric Bernstein operators. Firstly, we study the approximation behaviour of these operators including a local and global approximation results and the rate of approximation for the Lipschitz type space. The Voronovskaja type asymptotic formula and the rate of convergence of functions with derivatives of bounded variation are established. Finally, the theoretical results are demonstrated by using MAPLE software.
\end{abstract}

Keywords: Bernstein operators; rate of approximation; Voronovskaja type asymptotic formula

\section{Introduction}

A first fundamental result in approximation theory was Weierstrass approximation theorem [1] which forms the solid foundation of Approximation Theory. The proof of the theorem was quite long and difficult. So there were several proofs given by different famous mathematicians. One of them was given by Bernstein [2] which was easy and elegant, which also motivated the researchers to construct operators to deal with the approximation problems in different settings. Here, we give a Durrmeyer type generalization of parametric Bernstein operators. Let $C(j)$ be the space of all real valued continuous functions $\mathcal{S}$ on the interval $\jmath=[0,1]$. For $\mathcal{S} \in C(\jmath)$, Chen et al. [3] introduced a new family of generalized Bernstein operators depending upon a non-negative real parameter $0 \leq \theta \leq 1$, which is given as follows:

$$
T_{m}^{(\theta)}(\mathcal{S} ; x)=\sum_{s=0}^{m} p_{m, S}^{(\theta)}(x) \mathcal{S}\left(\frac{S}{m}\right), \quad x \in \jmath,
$$

where

$p_{m, s}^{(\theta)}(x)=\left[\left(\begin{array}{c}m-2 \\ s\end{array}\right)(1-\theta) x+\left(\begin{array}{c}m-2 \\ s-2\end{array}\right)(1-\theta)(1-x)+\left(\begin{array}{c}m \\ s\end{array}\right) \theta x(1-x)\right] x^{s-1}(1-x)^{m-s-1}$,

$m \geq 2, p_{1,0}^{(\theta)}(x)=1-x, p_{1,1}^{(\theta)}(x)=x$. For $\theta=1$, it reduces to original Bernstein operators.

Several types of such operators have been studied so far, for example, Kajla and Acar [4] gave the integral variant of the operators (1) and studied the approximation properties of these operators. Genuine Bernstein-Durrmeyer type operators were defined and studied in [5]. Abel and Heilmann [6] studied the complete asymptotic expansion of the Bernstein-Durrmeyer operators. Cárdenas-Morales and Gupta [7] considered a two-parameter family of summation-integral type operators involving 
Pólya-Eggenberger distribution. In 2015, Abel et al. [8] presented the Durrmeyer type modification of the Stancu operators and obtained some approximation theorems. Agrawal et al. [9] defined Stancu type Kantorovich modification of $q$-Bernstein-Schurer operators and studied some approximation theorems on uniform convergence as well as $A$-statistical convergence. Ansari et al. in [10] proposed Jakimovski-Leviatan-Durrmeyer type operators based on Appell polynomials and obtained some approximation results, e.g.,Voronovskaja type asymptotic formula, rate of convergence and weighted approximation of these operators. Acar et al. [11] presented a general class of linear positive operators and established Voronovskaya type theorems. In 2019, Mursaleen et al. [12] considered Stancu-Jakimovski-Leviatan-Durrmeyer type operators and studied simultaneous approximation and $A$-statistical approximation properties of these operators.

Acu and Kajla [13] established $\theta$-Bernstein operators depend on parameters $\rho_{1}, \rho_{2} \in \mathbb{N} \cup\{0\}$ as follows:

$$
\mathcal{B}_{m, \rho_{1}, \rho_{2}}^{(\theta)}(\mathcal{S} ; x)=\sum_{\mu=0}^{m-\rho_{1} \rho_{2}} p_{m-\rho_{1} \rho_{2}, \mu}^{(\theta)}(x) \sum_{s=0}^{\rho_{2}} p_{\rho_{2}, s}^{(\theta)}(x) \mathcal{S}\left(\frac{\mu+s \rho_{1}}{m}\right) .
$$

If $\rho_{1}=\rho_{2}=0$, these operators reduces to the operators $T_{m}^{(\theta)}$.

For $\mathcal{S} \in C(\jmath)$, we introduce a Durrmeyer type modification of the operators (2) as follows:

$$
U_{m, \rho_{1}, \rho_{2}}^{(\theta)}(\mathcal{S} ; x)=(m+1) \sum_{\mu=0}^{m-\rho_{1} \rho_{2}} p_{m-\rho_{1} \rho_{2}, \mu}^{(\theta)}(x) \sum_{s=0}^{\rho_{2}} p_{\rho_{2}, s}^{(\theta)}(x) \int_{0}^{1} p_{m, \mu+s \rho_{1}}(t) \mathcal{S}(t) d t .
$$

The aim of this paper is to derive approximation properties for the operators (3) by working on Korovkin's results [14]. We also compute the rate of convergence involving modulus of smoothness and Lipschitz type function.

\section{Auxiliary Results}

In this section, we derive some auxiliary results which will be used in proving our main results of subsequent sections. First, we determine moments and central moments for the operators (3).

Lemma 1. Let $e_{i}(t)=t^{i}, i=0,1,2 \ldots$. For the operators $U_{m, \rho_{1}, \rho_{2}}^{(\theta)}$, we have

(i) $U_{m, \rho_{1}, \rho_{2}}^{(\theta)}\left(e_{0} ; x\right)=1$;

(ii) $U_{m, \rho_{1}, \rho_{2}}^{(\theta)}\left(e_{1} ; x\right)=\frac{m x+1}{(m+2)}$;

(iii) $U_{m, \rho_{1}, \rho_{2}}^{(\theta)}\left(e_{2} ; x\right)=\frac{x^{2}\left(m^{2}-m-2+\rho_{1} \rho_{2}-\rho_{1}^{2}\left(\rho_{2}-2 \theta+2\right)+2 \theta\right)}{(m+3)(m+2)}$

$$
+\frac{x\left(2+4 m-\rho_{1} \rho_{2}+\rho_{1}^{2}\left(\rho_{2}-2 \theta+2\right)-2 \theta\right)}{(m+3)(m+2)}+\frac{2}{(m+3)(m+2)} ;
$$

(iv) $U_{m, \rho_{1}, \rho_{2}}^{(\theta)}\left(e_{3} ; x\right)=\frac{x^{3}}{(m+4)(m+3)(m+2)}\left[m^{3}-3 m^{2}-4 m+\rho_{1} \rho_{2}(3 m-2)+2 \rho_{1}^{3}\left(\rho_{2}-6 \theta+6\right)\right.$

$\left.-3 m \rho_{1}^{2}\left(2+\rho_{2}-2 \theta\right)-12(\theta-1)+6 m \theta\right]$

$+\frac{x^{2}}{(m+4)(m+3)(m+2)}\left[9 m^{2}+9 \rho_{1} \rho_{2}-3 m-3 m \rho_{1} \rho_{2}-3 \rho_{1}^{3}\left(6+\rho_{2}-6 \theta\right)-6 \rho_{1}^{2}\left(2+\rho_{2}-2 \theta\right)\right.$

$\left.+3 m \rho_{1}^{2}\left(2+\rho_{2}-2 \theta\right)+30(\theta-1)-6 m \theta\right]$

$+\frac{x\left(18 m-7 \rho_{1} \rho_{2}+\rho_{1}^{3}\left(\rho_{2}-6 \theta+6\right)+6 \rho_{1}^{2}\left(2+\rho_{2}-2 \theta\right)-18(\theta-1)\right)}{(m+4)(m+3)(m+2)}+\frac{6}{(m+4)(m+3)(m+2)} ;$

(v) $U_{m, \rho_{1}, \rho_{2}}^{(\theta)}\left(e_{4} ; x\right)=\frac{x^{4}}{(m+5)(m+4)(m+3)(m+2)}\left[54 m-m^{2}-6 m^{3}+m^{4}-6 \rho_{1} \rho_{2}-14 m \rho_{1} \rho_{2}+3 \rho_{1}^{2} \rho_{2}^{2}-6 \rho_{1}^{3} \rho_{2}^{2}+\right.$ $3 \rho_{1}^{4} \rho_{2}^{2}-6 m^{2} \rho_{1}\left(-\rho_{2}+\rho_{1}\left(2+\rho_{2}\right)\right)+8 m \rho_{1}^{3}\left(6+\rho_{2}-6 \theta\right)+6 \rho_{1}^{4} \rho_{2}(1-2 \theta)+6 m \rho_{1}^{2}\left(2+\rho_{2}-2 \theta\right)+72(-1+\theta)+$ 


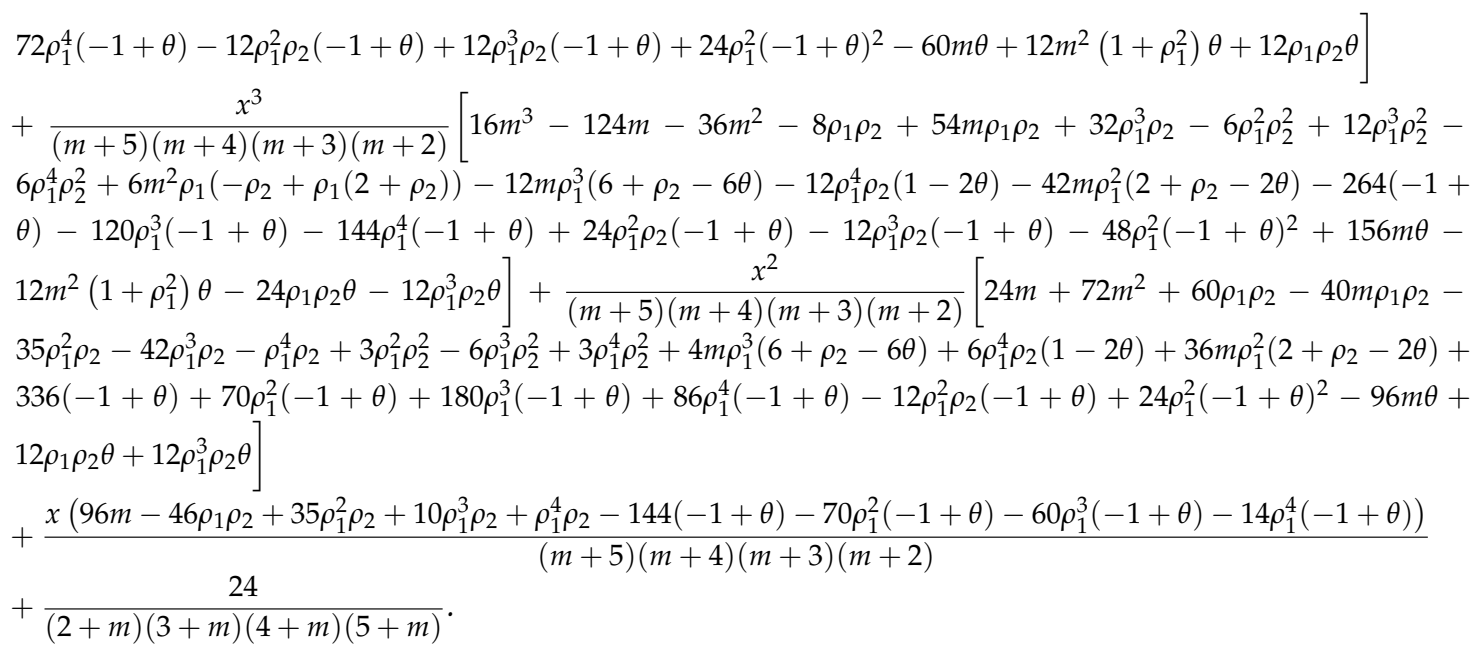

Let $\Theta_{m, \rho_{1}, \rho_{2}}^{(\theta), m}:=U_{m, \rho_{1}, \rho_{2}}^{(\theta)}\left((t-x)^{m} ; x\right), m=1,2,4$ be the central moments of $U_{m, \rho_{1}, \rho_{2}}^{(\theta)}$.

Lemma 2. For the operators $U_{m, \rho_{1}, \rho_{2}}^{(\theta)}$, we get

(i) $\Theta_{m, \rho_{1}, \rho_{2}}^{(\theta), 1}(x)=\left(\frac{1-2 x}{2+m}\right)$;

(ii) $\Theta_{m, \rho_{1}, \rho_{2}}^{(\theta), 2}(x)=\frac{2}{(2+m)(3+m)}+\frac{x\left(2 m-\rho_{1}\left(\rho_{2}-\rho_{1}\left(2+\rho_{2}-2 \theta\right)\right)-2(2+\theta)\right)}{(2+m)(3+m)}$

$+\frac{x^{2}\left(-2 m+\rho_{1}\left(\rho_{2}-\rho_{1}\left(2+\rho_{2}-2 \theta\right)\right)+2(2+\theta)\right)}{(2+m)(3+m)}$.

Lemma 3. For $m \in \mathbb{N}$, we have

$$
U_{m, \rho_{1}, \rho_{2}}^{(\theta)}\left((t-x)^{2} ; x\right) \leq \frac{\mathcal{W}_{\rho_{1}, \rho_{2}}^{(\theta)} x(1-x)}{(m+2)}=\delta_{m, \rho_{1}, \rho_{2}}^{(\theta)}(x), \forall x \in \jmath,
$$

where $\mathcal{W}_{\rho_{1}, \rho_{2}}^{(\theta)}$ is a positive constant depending on $\rho_{1}, \rho_{2}$ and $\theta$.

Proof. This lemma is established by direct computation and the details are missing.

Remark 1. For the operators $U_{m, \rho_{1}, \rho_{2}}^{(\theta)}$, we get

$$
\begin{aligned}
& \lim _{m \rightarrow \infty} m \Theta_{m, \rho_{1}, \rho_{2}}^{(\theta), 1}(x)=(1-2 x), \\
& \lim _{m \rightarrow \infty} m \Theta_{m, \rho_{1}, \rho_{2}}^{(\theta), 2}(x)=2 x(1-x), \\
& \lim _{m \rightarrow \infty} m^{2} \Theta_{m, \rho_{1}, \rho_{2}}^{(\theta), 4}(x)=12 x^{2}(1-x)^{2} .
\end{aligned}
$$

Lemma 4. For $\mathcal{S} \in C(\jmath)$, we have

$$
\left\|U_{m, \rho_{1}, \rho_{2}}^{(\theta)}(\mathcal{S} ; x)\right\| \leq\|\mathcal{S}\| .
$$

Proof. From Lemma 1 and Equation (3), we obtain

$$
\begin{aligned}
\left\|U_{m, \rho_{1}, \rho_{2}}^{(\theta)}\right\| & \leq(m+1) \sum_{\mu=0}^{m-\rho_{1} \rho_{2}} p_{m-\rho_{1} \rho_{2}, \mu}^{(\theta)}(x) \sum_{s=0}^{\rho_{2}} p_{\rho_{2}, s}^{(\theta)}(x) \int_{0}^{1} p_{m, \mu+s \rho_{1}}(t)|\mathcal{S}(t)| d t \\
& \leq\|\mathcal{S}\| U_{m, \rho_{1}, \rho_{2}}^{(\theta)}\left(e_{0} ; x\right)=\|\mathcal{S}\| .
\end{aligned}
$$


Theorem 1. Suppose that $\mathcal{S} \in C(\jmath)$. Show that $\lim _{m \rightarrow \infty} U_{m, \rho_{1}, \rho_{2}}^{(\theta)}(\mathcal{S} ; x)=\mathcal{S}(x)$, uniformly in 1 .

Proof. Since $U_{m, \rho_{1}, \rho_{2}}^{(\theta)}(1 ; x)=1, U_{m, \rho_{1}, \rho_{2}}^{(\theta)}(t ; x) \rightarrow x, U_{m, \rho_{1}, \rho_{2}}^{(\theta)}\left(t^{2} ; x\right) \rightarrow x^{2}$ as $m \rightarrow \infty$, uniformly in $j$. By Korovkin's results, it follows that $U_{m, p_{1}, \rho_{2}}^{(\theta)}(\mathcal{S} ; x)$ converges to $\mathcal{S}(x)$ uniformly on $\mathrm{f}$.

\section{Voronovskaja Type Theorems}

Here, we establish the Voronovskaja, Grüss-Voronovskaja type theorems and related results.

Theorem 2. Suppose that $\mathcal{S} \in C(j)$. If $\mathcal{S}^{\prime}, \mathcal{S}^{\prime \prime}$ exists at a point $x \in \jmath$ then

$$
\lim _{m \rightarrow \infty} m\left(U_{m, \rho_{1}, \rho_{2}}^{(\theta)}(\mathcal{S} ; x)-\mathcal{S}(x)\right)=(1-2 x) \mathcal{S}^{\prime}(x)+x(1-x) \mathcal{S}^{\prime \prime}(x),
$$

Further, if $\mathcal{S}^{\prime \prime} \in C(\jmath)$ then (4) holds uniformly in $\mathrm{j}$.

Proof. Applying the application of Taylor's theorem, we have

$$
\mathcal{S}(t)=\mathcal{S}(x)+(t-x) \mathcal{S}^{\prime}(x)+\frac{1}{2}(t-x)^{2} \mathcal{S}^{\prime \prime}(x)+\rho_{1}(t, x)(t-x)^{2},
$$

where $\rho_{1}(t, x) \rightarrow 0$ as $t \rightarrow x$ and is a continuous function on $\mathrm{j}$. Applying $U_{m, \rho_{1}, \rho_{2}}^{(\theta)}$ to (5), we get

$$
\begin{array}{r}
U_{m, \rho_{1}, \rho_{2}}^{(\theta)}(\mathcal{S} ; x)-\mathcal{S}(x)=\mathcal{S}^{\prime}(x) U_{m, \rho_{1}, \rho_{2}}^{(\theta)}((t-x) ; x)+\frac{1}{2} \mathcal{S}^{\prime \prime}(x) U_{m, \rho_{1}, \rho_{2}}^{(\theta)}\left((t-x)^{2} ; x\right)+U_{m, \rho_{1}, \rho_{2}}^{(\theta)}\left(\rho_{1}(t, x)(t-x)^{2} ; x\right), \\
\lim _{m \rightarrow \infty} m\left(U_{m, \rho_{1}, \rho_{2}}^{(\theta)}(\mathcal{S} ; x)-\mathcal{S}(x)\right)=(1-2 x) \mathcal{S}^{\prime}(x)+x(1-x) \mathcal{S}^{\prime \prime}(x)+\lim _{m \rightarrow \infty} m U_{m, \rho_{1}, \rho_{2}}^{(\theta)}\left(\rho_{1}(t, x)(t-x)^{2} ; x\right) .
\end{array}
$$

Since $\rho_{1}(t, x) \rightarrow 0$ as $t \rightarrow x$, for a given $\epsilon>0$, there exists $\delta>0$ such that $\left|\rho_{1}(t, x)\right|<\epsilon$ whenever $|t-x|<\delta$. For $|t-x| \geq \delta$, we have $\left|\rho_{1}(t, x)\right| \leq M \frac{(t-x)^{2}}{\delta^{2}}$, for some $M>0$. Let $\chi_{\delta}(t)$ denote the characteristic function of the interval $(x-\delta, x+\delta)$. In view of Remark 1 , we have

$$
\begin{aligned}
\left|U_{m, \rho_{1}, \rho_{2}}^{(\theta)}\left(\rho_{1}(t, x)(t-x)^{2} ; x\right)\right| & \leq U_{m, \rho_{1}, \rho_{2}}^{(\theta)}\left(\left|\rho_{1}(t, x)\right|(t-x)^{2} \chi_{\delta}(t) ; x\right)+U_{m, \rho_{1}, \rho_{2}}^{(\theta)}\left(\left|\rho_{1}(t, x)\right|(t-x)^{2}\left(1-\chi_{\delta}(t)\right) ; x\right) \\
& \left.<\epsilon U_{m, \rho_{1}, \rho_{2}}^{(\theta)}(t-x)^{2} ; x\right)+\frac{M}{\delta^{2}} U_{m, \rho_{1}, \rho_{2}}^{(\theta)}\left((t-x)^{4} ; x\right) \\
& =\epsilon O\left(\frac{1}{m}\right)+O\left(\frac{1}{m^{2}}\right) .
\end{aligned}
$$

which implies that $\lim _{m \rightarrow \infty} m U_{m, \rho_{1}, \rho_{2}}^{(\theta)}\left(\rho_{1}(t, x)(t-x)^{2} ; x\right)=0$, due to the arbitrariness of $\epsilon>0$. This complete the first half of the theorem.

To show the uniformity postulation, by the definition of uniformly continuity of $\mathcal{S}$ in $]$, the $\delta$ must be independent of $x$ and all the other estimates hold uniformly in $x \in \jmath$.

In [15], Acar et al. obtained a Grüss type approximation result and a Grüss-Voronovskaja-type result for linear and positive operators. Many authors have established in this direction so that we refer the authors to [16-18] and references therein.

The next result is the Grüss-Voronovskaja type theorem for $U_{m, \rho_{1}, \rho_{2}}^{(\theta)}$.

Theorem 3. Let $\mathcal{S}, \hbar \in C^{2}(\jmath)$. Then, for each $x \in \jmath$,

$$
\lim _{m \rightarrow \infty} m\left\{U_{m, \rho_{1}, \rho_{2}}^{(\theta)}((\mathcal{S} \hbar) ; x)-U_{m, \rho_{1}, \rho_{2}}^{(\theta)}(\mathcal{S} ; x) U_{m, \rho_{1}, \rho_{2}}^{(\theta)}(\hbar ; x)\right\}=\mathcal{S}^{\prime}(x) \hbar^{\prime}(x) 2 x(1-x) .
$$


Proof. The following relation holds

$$
\begin{aligned}
& U_{m, p_{1}, \rho_{2}}^{(\theta)}(\mathcal{S} \hbar ; x)-U_{m, p_{1}, \rho_{2}}^{(\theta)}(\mathcal{S} ; x) U_{m, \rho_{1}, \rho_{2}}^{(\theta)}(\hbar ; x)=U_{m, p_{1}, \rho_{2}}^{(\theta)}(\mathcal{S} \hbar ; x)-\mathcal{S}(x) \hbar(x)-(\mathcal{S} \hbar)^{\prime}(x) \Theta_{m, \rho_{1}, \rho_{2}}^{(\theta), 1}(x)-\frac{1}{2}(\mathcal{S} \hbar)^{\prime \prime}(x) \Theta_{m, p_{1}, \rho_{2}}^{(\theta), 2}(x) \\
& -\hbar(x)\left\{U_{m, \rho_{1}, \rho_{2}}^{(\theta)}(\mathcal{S} ; x)-\mathcal{S}(x)-\mathcal{S}^{\prime}(x) \Theta_{m, \rho_{1}, \rho_{2}}^{(\theta), 1}(x)-\frac{1}{2} \mathcal{S}^{\prime \prime}(x) \Theta_{m, \rho_{1}, \rho_{2}}^{(\theta), 2}(x)\right\} \\
& -U_{m, \rho_{1}, \rho_{2}}^{(\theta)}(S ; x)\left\{U_{m, p_{1}, \rho_{2}}^{(\theta)}(\hbar ; x)-\hbar(x)-\hbar^{\prime}(x) \Theta_{m, \rho_{1}, \rho_{2}}^{(\theta), 1}(x)-\frac{1}{2} \hbar^{\prime \prime}(x) \Theta_{m, p_{1}, \rho_{2}}^{(\theta), 2}(x)\right\} \\
& +\frac{1}{2} \Theta_{m, \rho_{1}, \rho_{2}}^{(\theta), 2}(x)\left\{\mathcal{S}(x) \hbar^{\prime \prime}(x)+2 \mathcal{S}^{\prime}(x) \hbar^{\prime}(x)-\hbar^{\prime \prime}(x) U_{m, p_{1}, \rho_{2}}^{(\theta)}(\mathcal{S} ; x)\right\}+\Theta_{m, \rho_{1}, \rho_{2}}^{(\theta), 1}(x)\left\{\mathcal{S}(x) \hbar^{\prime}(x)-\hbar^{\prime}(x) U_{m, \rho_{1}, \rho_{2}}^{(\theta)}(\mathcal{S} ; x)\right\} .
\end{aligned}
$$

Now, by using Theorem 1, Theorem 2 and Remark 1, we get

$$
\begin{aligned}
& \lim _{m \rightarrow \infty} m\left\{U_{m, \rho_{1}, \rho_{2}}^{(\theta)}(\mathcal{S} \hbar ; x)-U_{m, \rho_{1}, \rho_{2}}^{(\theta)}(\mathcal{S} ; x) U_{m, \rho_{1}, \rho_{2}}^{(\theta)}(\hbar ; x)\right\} \\
& =\lim _{m \rightarrow \infty} m \mathcal{S}^{\prime}(x) \hbar^{\prime}(x) \Theta_{m, \rho_{1}, \rho_{2}}^{(\theta), 2}(x)+\lim _{m \rightarrow \infty} \frac{1}{2} m \hbar^{\prime \prime}(x)\left\{\mathcal{S}(x)-U_{m, \rho_{1}, \rho_{2}}^{(\theta)}(\mathcal{S} ; x)\right\} \Theta_{m, \rho_{1}, \rho_{2}}^{(\theta),}(x) \\
& +\lim _{m \rightarrow \infty} m \hbar^{\prime}(x)\left\{\mathcal{S}(x)-U_{m, \rho_{1}, \rho_{2}}^{(\theta)}(\mathcal{S} ; x)\right\} \Theta_{m, \rho_{1}, \rho_{2}}^{(\theta), 1}(x)=\mathcal{S}^{\prime}(x) \hbar^{\prime}(x) 2 x(1-x) .
\end{aligned}
$$

Lipschitz-type space with two parameters $\alpha_{1} \geq 0, \alpha_{2}>0$ is defined in [19] as below:

$$
\operatorname{Lip}_{M}^{\left(\alpha_{1}, \alpha_{2}\right)}(\sigma):=\left\{\mathcal{S} \in C(\jmath):|\mathcal{S}(t)-\mathcal{S}(x)| \leq M \frac{|t-x|^{\sigma}}{\left(t+\alpha_{1} x^{2}+\alpha_{2} x\right)^{\frac{\sigma}{2}}} ; t \in \jmath, x \in(0,1]\right\},
$$

where $0<\sigma \leq 1$.

Theorem 4. Suppose that $\mathcal{S} \in \operatorname{Lip}_{M}^{\left(\alpha_{1}, \alpha_{2}\right)}(\sigma)$. Prove that

$$
\left|U_{m, \rho_{1}, \rho_{2}}^{(\theta)}(\mathcal{S} ; x)-\mathcal{S}(x)\right| \leq M\left(\frac{\Theta_{m, \rho_{1}, \rho_{2}}^{(\theta), 2}(x)}{\alpha_{1} x^{2}+\alpha_{2} x}\right)^{\sigma / 2}, \forall x \in(0,1] .
$$

Proof. Using the application of Holder's inequality and Lemma 2, we may write

$$
\begin{aligned}
\left|U_{m, \rho_{1}, \rho_{2}}^{(\theta)}(\mathcal{S} ; x)-\mathcal{S}(x)\right| \leq & (m+1) \sum_{\mu=0}^{m-\rho_{1} \rho_{2}} p_{m-\rho_{1} \rho_{2}, \mu}^{(\theta)}(x) \sum_{s=0}^{\rho_{2}} p_{\rho_{2}, s}^{(\theta)}(x) \int_{0}^{1}|\mathcal{S}(t)-\mathcal{S}(x)| p_{m, \mu+s \rho_{1}}(t) d t \\
\leq & (m+1) \sum_{\mu=0}^{m-\rho_{1} \rho_{2}} p_{m-\rho_{1} \rho_{2}, \mu}^{(\theta)}(x) \sum_{s=0}^{\rho_{2}} p_{\rho_{2}, s}^{(\theta)}(x)\left(\int_{0}^{1}|\mathcal{S}(t)-\mathcal{S}(x)|^{\frac{2}{\sigma}} p_{m, \mu+s \rho_{1}}(t) d t\right)^{\frac{\sigma}{2}} \\
\leq & \left\{(m+1) \sum_{\mu=0}^{m-\rho_{1} \rho_{2}} p_{m-\rho_{1} \rho_{2}, \mu}^{(\theta)}(x) \sum_{s=0}^{\rho_{2}} p_{\rho_{2}, s}^{(\theta)}(x) \int_{0}^{1}|\mathcal{S}(t)-\mathcal{S}(x)|^{\frac{2}{\sigma}} p_{m, \mu+s \rho_{1}}(t) d t\right\}^{\frac{\sigma}{2}} \\
& \times\left((m+1) \sum_{\mu=0}^{m-\rho_{1} \rho_{2}} p_{m-\rho_{1} \rho_{2}, \mu}^{(\theta)}(x) \sum_{s=0}^{\rho_{2}} p_{\rho_{2}, s}^{(\theta)}(x) \int_{0}^{1} p_{m, \mu+s \rho_{1}}(t) d t\right)^{\frac{2-\sigma}{2}} \\
= & \left((m+1) \sum_{\mu=0}^{m-\rho_{1} \rho_{2}} p_{m-\rho_{1} \rho_{2}, \mu}^{(\theta)}(x) \sum_{s=0}^{\rho_{2}} p_{\rho_{2}, s}^{(\theta)}(x) \int_{0}^{1}|\mathcal{S}(t)-\mathcal{S}(x)|^{\frac{2}{\sigma}} p_{m, \mu+s \rho_{1}}(t) d t\right)^{\frac{\sigma}{2}} \\
\leq & M\left((m+1) \sum_{\mu=0}^{m-\rho_{1} \rho_{2}} p_{m-\rho_{1} \rho_{2}, \mu}^{(\theta)}(x) \sum_{s=0}^{\rho_{2}} p_{\rho_{2}, s}^{(\theta)}(x) \int_{0}^{1} \frac{(t-x)^{2}}{\left(t+\alpha_{1} x^{2}+\alpha_{2} x\right)} p_{m, \mu+s \rho_{1}}(t) d t\right)^{\frac{\sigma}{2}} \\
\leq & \frac{M}{\left(\alpha_{1} x^{2}+\alpha_{2} x\right)^{\frac{\sigma}{2}}}\left((m+1) \sum_{\mu=0}^{m-\rho_{1} \rho_{2}} p_{m-\rho_{1} \rho_{2}, \mu}^{(\theta)}(x) \sum_{s=0}^{\rho_{2}} p_{\rho_{2}, s}^{(\theta)}(x) \int_{0}^{1}(t-x)^{2} p_{m, \mu+s \rho_{1}}(t) d t\right)^{\frac{\sigma}{2}} \\
= & \frac{M}{\left(\alpha_{1} x^{2}+\alpha_{2} x\right)^{\frac{\sigma}{2}}} U_{m, \rho_{1}, \rho_{2}}^{(\theta)}\left((t-x)^{2} ; x\right)^{\frac{\sigma}{2}} \\
= & \frac{M}{\left(\alpha_{1} x^{2}+\alpha_{2} x\right)^{\frac{\sigma}{2}}}\left(\Theta_{m, \rho_{1}, \rho_{2}}^{(\theta), 2}(x)\right)^{\frac{\sigma}{2}}
\end{aligned}
$$


Theorem 5. For $\mathcal{S} \in C^{1}(\jmath)$ and $x \in$, we have

$$
\left|U_{m, \rho_{1}, \rho_{2}}^{(\theta)}(\mathcal{S} ; x)-\mathcal{S}(x)\right| \leq\left|\frac{1-2 x}{(m+2)}\right|\left|\mathcal{S}^{\prime}(x)\right|+2 \sqrt{\Theta_{m, \rho_{1}, \rho_{2}}^{(\theta), 2}(x)} \omega\left(\mathcal{S}^{\prime}, \sqrt{\Theta_{m, \rho_{1}, \rho_{2}}^{(\theta), 2}(x)}\right) .
$$

Proof. Let $\mathcal{S} \in C^{1}(\jmath)$. For any $t, x \in \jmath$, we have

$$
\mathcal{S}(t)-\mathcal{S}(x)=\mathcal{S}^{\prime}(x)(t-x)+\int_{x}^{t}\left(\mathcal{S}^{\prime}(u)-\mathcal{S}^{\prime}(x)\right) d u .
$$

Using $U_{m, \rho_{1}, \rho_{2}}^{(\theta)}(\cdot ; x)$ on both sides of the above relation, we have

$$
U_{m, \rho_{1}, \rho_{2}}^{(\theta)}\left(\mathcal{S}(t)-\mathcal{S}(x) ; q_{m}, x\right)=\mathcal{S}^{\prime}(x) U_{m, \rho_{1}, \rho_{2}}^{(\theta)}(t-x ; x)+U_{m, \rho_{1}, \rho_{2}}^{(\theta)}\left(\int_{x}^{t}\left(\mathcal{S}^{\prime}(u)-\mathcal{S}^{\prime}(x)\right) d u ; x\right)
$$

Applying $|\mathcal{S}(t)-\mathcal{S}(x)| \leq \omega(\mathcal{S}, \delta)\left(\frac{|t-x|}{\delta}+1\right), \delta>0$, we have

$$
\left|\int_{x}^{t}\left(\mathcal{S}^{\prime}(u)-\mathcal{S}^{\prime}(x)\right) d u\right| \leq \omega\left(\mathcal{S}^{\prime}, \delta\right)\left(\frac{(t-x)^{2}}{\delta}+|t-x|\right)
$$

it follows that

$$
\left|U_{m, \rho_{1}, \rho_{2}}^{(\theta)}(\mathcal{S} ; x)-\mathcal{S}(x)\right| \leq\left|\mathcal{S}^{\prime}(x)\right| \quad\left|U_{m, \rho_{1}, \rho_{2}}^{(\theta)}(t-x ; x)\right|+\omega\left(\mathcal{S}^{\prime}, \delta\right)\left\{\frac{1}{\delta} U_{m, \rho_{1}, \rho_{2}}^{(\theta)}\left((t-x)^{2} ; x\right)+U_{m, \rho_{1}, \rho_{2}}^{(\theta)}(|t-x| ; x)\right\} .
$$

Applying Cauchy-Schwarz inequality, we get

$$
\begin{aligned}
\left|U_{m, \rho_{1}, \rho_{2}}^{(\theta)}(\mathcal{S} ; x)-\mathcal{S}(x)\right| \leq & \left|\mathcal{S}^{\prime}(x)\right|\left|U_{m, \rho_{1}, \rho_{2}}^{(\theta)}(t-x ; x)\right| \\
& +\omega\left(\mathcal{S}^{\prime}, \delta\right)\left\{\frac{1}{\delta} \sqrt{U_{m, \rho_{1}, \rho_{2}}^{(\theta)}\left((t-x)^{2} ; x\right)}+1\right\} \sqrt{U_{m, \rho_{1}, \rho_{2}}^{(\theta)}\left((t-x)^{2} ; x\right)} .
\end{aligned}
$$

Now, taking $\delta=\sqrt{\Theta_{m, p_{1}, \rho_{2}}^{(\theta), 2}(x)}$, we get (6).

\section{Local Approximation}

In this section, we study the local approximation property for our operators with the help of $K$-functional.

The $K$-functional is given by:

$$
K_{2}(\mathcal{S}, \delta)=\inf \left\{\|\mathcal{S}-\hbar\|+\delta\left\|\hbar^{\prime \prime}\right\|: \hbar \in W^{2}\right\}(\delta>0),
$$

where $W^{2}=\left\{\hbar: \hbar^{\prime \prime} \in C(\jmath)\right\}$ and uniform norm on $C(\jmath)$ is denoted by $\|$.$\| . By [20] there will be a$ positive constant $M>0$ such that

$$
K_{2}(\mathcal{S}, \delta) \leq M \omega_{2}(\mathcal{S}, \sqrt{\delta})
$$

where the second order modulus of continuity for $\mathcal{S} \in C(j)$ is defined as

$$
\omega_{2}(\mathcal{S}, \sqrt{\delta})=\sup _{0<h \leq \sqrt{\delta} x, x+2 h \in j} \sup _{0<h}|\mathcal{S}(x+2 h)-2 \mathcal{S}(x+h)+\mathcal{S}(x)| .
$$


We define the usual modulus of continuity for $\mathcal{S} \in C(\jmath)$ as

$$
\omega(\mathcal{S}, \delta)=\sup _{0<h \leq \delta x, x+h \in j} \sup _{0}|\mathcal{S}(x+h)-\mathcal{S}(x)| .
$$

Theorem 6. For the operators $U_{m, \rho_{1}, \rho_{2}}^{(\theta)}$, there exists a constant $M>0$ such that

$$
\left|U_{m, \rho_{1}, \rho_{2}}^{(\theta)}(\mathcal{S} ; x)-\mathcal{S}(x)\right| \leq M \omega_{2}\left(\mathcal{S},(m+2)^{-1 / 2} \sqrt{\delta_{m, \rho_{1}, \rho_{2}}^{(\theta)}}(x)\right)+\omega\left(\mathcal{S},\left|\frac{1-2 x}{m+2}\right|\right)
$$

where $\mathcal{S} \in C(\jmath), \theta \in \jmath, \delta_{m, \rho_{1}, \rho_{2}}^{(\theta)}(x)=\varphi^{2}(x)+\frac{1}{(m+2)}$ and $x \in \jmath$.

Proof. We define the auxiliary operators as follows:

$$
\bar{U}_{m, \rho_{1}, \rho_{2}}^{(\theta)}(\mathcal{S} ; x)=U_{m, \rho_{1}, \rho_{2}}^{(\theta)}(\mathcal{S} ; x)+\mathcal{S}(x)-\mathcal{S}\left(\frac{m x+1}{m+2}\right) .
$$

Then, we can easily check that

$$
\bar{U}_{m, \rho_{1}, \rho_{2}}^{(\theta)}(1 ; x)=1 \quad \text { and } \quad \bar{U}_{m, \rho_{1}, \rho_{2}}^{(\theta)}(t ; x)=x .
$$

By the application of Taylor's theorem and taking $t \in \jmath$ and $\hbar \in W^{2}$, we get

$$
\hbar(t)=\hbar(x)+(t-x) \hbar^{\prime}(x)+\int_{x}^{t}(t-u) \hbar^{\prime \prime}(u) d u .
$$

The operator $\bar{U}_{m, \rho_{1}, \rho_{2}}^{(\theta)}$ is applied in the above equation on both sides, we obtain

$$
\begin{aligned}
\bar{U}_{m, \rho_{1}, \rho_{2}}^{(\theta)}(\hbar ; x) & =\hbar(x)+\bar{U}_{m, \rho_{1}, \rho_{2}}^{(\theta)}\left(\int_{x}^{t}(t-u) \hbar^{\prime \prime}(u) d u\right) \\
& =\hbar(x)+U_{m, \rho_{1}, \rho_{2}}^{(\theta)}\left(\int_{x}^{t}(t-u) \hbar^{\prime \prime}(u) d u, x\right)-\int_{x}^{\frac{m x+1}{(m+2)}}\left(\frac{m x+1}{m+2}-u\right) \hbar^{\prime \prime}(u) d u .
\end{aligned}
$$

Hence

$$
\begin{aligned}
& \left|\bar{U}_{m, \rho_{1}, \rho_{2}}^{(\theta)}(\hbar ; x)-\hbar(x)\right| \leq U_{m, \rho_{1}, \rho_{2}}^{(\theta)}\left(\left|\int_{x}^{t}\right| t-u|| \hbar^{\prime \prime}(u)|d u|, x\right)+\left|\int_{x}^{\frac{m x+1}{m+2)}}\right| \frac{m x+1}{m+2}-u|| \hbar^{\prime \prime}(u)|d u| \\
& \leq\left\{U_{m, \rho_{1}, \rho_{2}}^{(\theta)}\left((t-x)^{2} ; x\right)+\left(\frac{m x+1}{m+2}-x\right)^{2}\right\}\left\|\hbar^{\prime \prime}\right\| \\
& =\left\{U_{m, \rho_{1}, \rho_{2}}^{(\theta)}\left((t-x)^{2} ; x\right)+\left(\frac{1-2 x}{m+2}\right)^{2}\right\}\left\|\hbar^{\prime \prime}\right\| \text {. }
\end{aligned}
$$

From Lemma 3, we have

$$
\begin{aligned}
U_{m, \rho_{1}, \rho_{2}}^{(\theta)}\left((t-x)^{2} ; x\right)+\left(\frac{1-2 x}{m+2}\right)^{2} & \leq \frac{2}{(m+2)} \delta_{m, \rho_{1}, \rho_{2}}^{(\theta)}(x)+\left(\frac{1-2 x}{m+2}\right)^{2} \\
& \leq \frac{2}{(m+2)} \delta_{m, \rho_{1}, \rho_{2}}^{(\theta)}(x)+\frac{1}{(m+2)^{2}} \\
& \leq \frac{3}{(m+2)} \delta_{m, \rho_{1}, \rho_{2}}^{(\theta)}(x) .
\end{aligned}
$$


Thus, by (8) we have

$$
\left|\bar{U}_{m, \rho_{1}, \rho_{2}}^{(\theta)}(\hbar ; x)-\hbar(x)\right| \leq \frac{3}{(m+2)} \delta_{m, \rho_{1}, \rho_{2}}^{(\theta)}(x)|| \hbar^{\prime \prime} \|,
$$

where $x \in \jmath$. Furthermore, by Lemma 4, we have

$$
\left|\bar{U}_{m, \rho_{1}, \rho_{2}}^{(\theta)}(\mathcal{S} ; x)\right| \leq 3|| \mathcal{S} \|
$$

for all $\mathcal{S} \in C(\jmath)$ and $x \in \jmath$.

Now, for $\mathcal{S} \in C(\jmath)$ and $\hbar \in W^{2}$, using (10) and (11) we obtain that

$$
\begin{aligned}
\left|U_{m, \rho_{1}, \rho_{2}}^{(\theta)}(\mathcal{S} ; x)-\mathcal{S}(x)\right| & \leq\left|\bar{U}_{m, \rho_{1}, \rho_{2}}^{(\theta)}(\mathcal{S} ; x)-\mathcal{S}(x)+\mathcal{S}\left(\frac{m x+1}{m+2}\right)-\mathcal{S}(x)\right| \\
& \leq\left|\bar{U}_{m, \rho_{1}, \rho_{2}}^{(\theta)}(\mathcal{S}-\hbar ; x)\right|+\left|\bar{U}_{m, \rho_{1}, \rho_{2}}^{(\theta)}(\hbar ; x)-\hbar(x)\right|+|\hbar(x)-\mathcal{S}(x)| \\
& +\left|\mathcal{S}\left(\frac{m x+1}{m+2}\right)-\mathcal{S}(x)\right| \\
& \leq 4|| \mathcal{S}-\hbar||+\frac{3}{(m+2)} \delta_{m, \rho_{1}, \rho_{2}}^{(\theta)}(x)|| \hbar^{\prime \prime}||+\omega\left(\mathcal{S},\left|\frac{1-2 x}{m+2}\right|\right) .
\end{aligned}
$$

Using the property of infimum on the right hand side over all $\hbar \in W^{2}$, we have

$$
\left|U_{m, \rho_{1}, \rho_{2}}^{(\theta)}(\mathcal{S} ; x)-\mathcal{S}(x)\right| \leq 4 K_{2}\left(\mathcal{S}, \frac{1}{(m+2)} \delta_{m, \rho_{1}, \rho_{2}}^{(\theta)}(x)\right)+\omega\left(\mathcal{S},\left|\frac{1-2 x}{m+2}\right|\right) .
$$

Now by examining the relation (7), we get

$$
\left|U_{m, \rho_{1}, \rho_{2}}^{(\theta)}(\mathcal{S} ; x)-\mathcal{S}(x)\right| \leq M \omega_{2}\left(\mathcal{S},(m+2)^{-1 / 2} \sqrt{\delta_{m, \rho_{1}, \rho_{2}}^{(\theta)}}(x)\right)+\omega\left(\mathcal{S},\left|\frac{1-2 x}{m+2}\right|\right) .
$$

\section{Global Approximation}

The following result provides the global approximation using the modulus of continuity of Ditzian-Totik and the related $K$-functional.

Suppose that $\mathcal{S} \in C(\jmath)$ and $\varphi(x)$ is defined as $\sqrt{x(1-x)}, x \in \jmath$. The second order modulus of continuity which is given by Ditzian-Totik

$$
\omega_{2}^{\varphi}(\mathcal{S}, \sqrt{\delta})=\sup _{0<h \leq \sqrt{\delta}} \sup _{x \pm h \varphi(x) \in j}|\mathcal{S}(x+h \varphi(x))-2 \mathcal{S}(x)+\mathcal{S}(x-h \varphi(x))|,
$$

and related $K$-functional is defined as,

$$
\tilde{K}_{2, \varphi(x)}(\mathcal{S}, \delta)=\inf \left\{\|\mathcal{S}-\hbar\|+\delta\left\|\varphi^{2} \hbar^{\prime \prime}\right\|+\delta^{2}\left\|\hbar^{\prime \prime}\right\|: \hbar \in W^{2}(\varphi)\right\},(\delta>0),
$$

where $W^{2}(\varphi)=\left\{\hbar \in C(j): \hbar^{\prime} \in A C_{l o c}, \varphi^{2} \hbar^{\prime \prime} \in C(j)\right\}$ and $\hbar^{\prime} \in A C_{l o c}$ means that $\hbar$ is derivable and $\hbar^{\prime}$ is absolutely continuous on every closed interval $[a, b] \subset(0,1)$. By ([21],Theorem 1.3.1) we can say that $\exists M>0$, such that

$$
\tilde{K}_{2, \varphi(x)}(\mathcal{S}, \delta) \leq M \omega_{2}^{\varphi}(\mathcal{S}, \sqrt{\delta})
$$


The first order Ditzian-Totik modulus is defined as

$$
\overrightarrow{\omega_{\psi}}(\mathcal{S}, \delta)=\sup _{0<h \leq \delta} \sup _{x \pm \frac{h}{2} \psi(x) \in j}\left|\mathcal{S}\left(x+\frac{h}{2} \psi(x)\right)-\mathcal{S}\left(x-\frac{h}{2} \psi(x)\right)\right|,
$$

where $\psi: \jmath \rightarrow \mathbb{R}$ is an admissible step-weight function.

Now we state our next main theorem.

Theorem 7. Let $\mathcal{S} \in C(\jmath)$ and $0 \leq \theta \leq 1$. Then, for $x \in \jmath$,

$$
\left\|U_{m, \rho_{1}, \rho_{2}}^{(\theta)} \mathcal{S}-\mathcal{S}\right\| \leq M \omega_{2}^{\varphi}\left(\mathcal{S},(m+2)^{-1 / 2}\right)+\overrightarrow{\omega_{\psi}}\left(\mathcal{S},(m+2)^{-1}\right)+\omega\left(\mathcal{S} ;(m+2)^{-1}\right)
$$

where $\varphi^{2}(x)=x(1-x)$ and $\psi(x)= \begin{cases}1-2 x & x \in[0,1 / 2] \\ 2 x-1 & x \in[1 / 2,1]\end{cases}$

Proof. The auxiliary operators is considered as

$$
\bar{U}_{m, \rho_{1}, \rho_{2}}^{(\theta)}(\mathcal{S} ; x)=U_{m, \rho_{1}, \rho_{2}}^{(\theta)}(\mathcal{S} ; x)+\mathcal{S}(x)-\mathcal{S}\left(\frac{m x+1}{m+2}\right) .
$$

Let $\hbar \in W^{2}(\varphi)$ then by expanding $\hbar$ using Taylor's theorem and as given in the proof of Theorem 6, we get

$$
\left|\bar{U}_{m, \rho_{1}, \rho_{2}}^{(\theta)}(\hbar ; x)-\hbar(x)\right| \leq U_{m, \rho_{1}, \rho_{2}}^{(\theta)}\left(\left|\int_{x}^{t}\right| t-u|| \hbar^{\prime \prime}(u)|d u|, x\right)+\int_{x}^{\frac{m x+1}{(m+2)}}\left|\frac{m x+1}{m+2}-u\right|\left|\hbar^{\prime \prime}(u)\right| d u .
$$

Setting $u=\beta x+(1-\beta) t, \beta \in \jmath$, and also applying the concavity of $\delta_{m, \rho_{1}, \rho_{2}}^{(\theta)}$, we have

$$
\frac{|t-u|}{\delta_{m, \rho_{1}, \rho_{2}}^{(\theta)}(u)}=\frac{\beta|t-x|}{\delta_{m, \rho_{1}, \rho_{2}}^{(\theta)}(\beta x+(1-\beta) t)} \leq \frac{\beta|t-x|}{\delta_{m, \rho_{1}, \rho_{2}}^{(\theta)}(x) \beta+\delta_{m, \rho_{1}, \rho_{2}}^{(\theta)}(t)(1-\beta)} \leq \frac{|t-x|}{\delta_{m, \rho_{1}, \rho_{2}}^{(\theta)}(x)} .
$$

Thus, using (14) in the inequality (13)

$$
\begin{aligned}
\left|\bar{U}_{m, p_{1}, \rho_{2}}^{(\theta)}(\hbar ; x)-\hbar(x)\right| & \leq U_{m, \rho_{1}, \rho_{2}}^{(\theta)}\left(\left|\int_{x}^{t} \frac{|t-u|}{\delta_{m, \rho_{1}, p_{2}}^{(\theta)}(u)} d u\right|, x\right)\left\|\delta_{m, p_{1}, \rho_{2}}^{(\theta)} \hbar^{\prime \prime}\right\|+\left(\int_{x}^{\frac{m x+1}{(m+2)}} \frac{\left|\frac{m x+1}{m+2}-u\right|}{\delta_{m, p_{1}, \rho_{2}}^{(\theta)}(u)} d u\right)\left\|\delta_{m, \rho_{1}, \rho_{2}}^{(\theta)} \hbar^{\prime \prime}\right\| . \\
& \leq \frac{1}{\delta_{m, p_{1}, \rho_{2}}^{(\theta)}(x)}\left\|\delta_{m, p_{1}, \rho_{2}}^{(\theta)} \hbar^{\prime \prime}\right\|\left[U_{m, \rho_{1}, \rho_{2}}^{(\theta)}\left((t-x)^{2} ; x\right)+\left(\frac{1-2 x}{m+2}\right)^{2}\right] .
\end{aligned}
$$

Now, using the inequality (9), we get

$$
\begin{aligned}
\left|\bar{U}_{m, \rho_{1}, \rho_{2}}^{(\theta)}(\hbar ; x)-\hbar(x)\right| & \leq \frac{3}{(m+2)}\left\|\delta_{m, \rho_{1}, \rho_{2}}^{(\theta)} \hbar^{\prime \prime}\right\| \\
& \leq \frac{3}{(m+2)}\left(\left\|\varphi^{2} \hbar^{\prime \prime}\right\|+\frac{1}{(m+2)}\left\|\hbar^{\prime \prime}\right\|\right) .
\end{aligned}
$$

Applying (11) and (15), we have for $\mathcal{S} \in C(\jmath)$,

$$
\begin{aligned}
\left|U_{m, \rho_{1}, \rho_{2}}^{(\theta)}(\mathcal{S} ; x)-\mathcal{S}(x)\right| & \leq\left|\bar{U}_{m, \rho_{1}, \rho_{2}}^{(\theta)}(\mathcal{S}-\hbar, x)\right|+\left|\bar{U}_{m, \rho_{1}, \rho_{2}}^{(\theta)}(\hbar ; x)-\hbar(x)\right|+|\hbar(x)-\mathcal{S}(x)| \\
& +\left|\mathcal{S}\left(\frac{m x+1}{m+2}\right)-\mathcal{S}(x)\right| \\
& \leq 4|| \mathcal{S}-\hbar||+\frac{3}{(m+2)}|| \varphi^{2} \hbar^{\prime \prime}||+\frac{3}{(m+2)^{2}}|| \hbar^{\prime \prime}||+\left|\mathcal{S}\left(\frac{m x+1}{m+2}\right)-\mathcal{S}(x)\right|
\end{aligned}
$$


For all $\hbar \in W^{2}(\varphi)$ using the property of infimum on the right hand side, we have

$$
\left|U_{m, \rho_{1}, \rho_{2}}^{(\theta)}(\mathcal{S} ; x)-\mathcal{S}(x)\right| \leq 4 \tilde{K}_{2, \varphi}\left(\mathcal{S}, \frac{1}{m+2}\right)+\left|\mathcal{S}\left(\frac{m x+1}{m+2}\right)-\mathcal{S}(x)\right|
$$

Also,

$$
\begin{aligned}
\left|\mathcal{S}\left(\frac{m x+1}{m+2}\right)-\mathcal{S}(x)\right| & =\left|\mathcal{S}\left(x+\frac{1-2 x}{m+2}\right)-\mathcal{S}(x)\right| \\
& \leq\left|\mathcal{S}\left(x+\frac{(1-2 x)}{m+2}\right)-\mathcal{S}\left(x-\frac{(1-2 x)}{m+2}\right)\right|+\left|\mathcal{S}\left(x-\frac{(1-2 x)}{m+2}\right)-\mathcal{S}(x)\right| \\
& \leq \overrightarrow{\omega_{\psi}}\left(\mathcal{S},(m+2)^{-1}\right)+\omega\left(\mathcal{S} ;(m+2)^{-1}\right) .
\end{aligned}
$$

Hence, combining (12), (16) and (17), the desired relation is immediate.

\section{Rate of Approximation}

In this section, we study the rate of convergence of functions with derivatives of bounded variation.

The class of all absolutely continuous functions $\mathcal{S}$ is denoted by $D B V_{(\jmath)}$, defined and having a derivative $\mathcal{S}^{\prime}$ on $\mathrm{j}$, analogous to a bounded variation function on $\mathrm{j}$.

The representation of functions $\mathcal{S} \in D B V_{(\jmath)}$ is

$$
\mathcal{S}(x)=\int_{0}^{x} \hbar(t) d t+\mathcal{S}(0)
$$

where $\hbar$ is a bounded variation function on $j$.

The operators $U_{m, \rho_{1}, \rho_{2}}^{(\theta)}(\mathcal{S} ; x)$ also admit the integral representation

$$
U_{m, \rho_{1}, \rho_{2}}^{(\theta)}(\mathcal{S} ; x)=\int_{0}^{1} \mathcal{N}_{m, \rho_{1}, \rho_{2}}^{(\theta)}(x, t) \mathcal{S}(t) d t
$$

where the kernel $\mathcal{N}_{m, p_{1}, \rho_{2}}^{(\theta)}(x, t)$ is given by

$$
\mathcal{N}_{m, \rho_{1}, \rho_{2}}^{(\theta)}(x, t)=(m+1) \sum_{\mu=0}^{m-\rho_{1} \rho_{2}} p_{m-\rho_{1} \rho_{2}, \mu}^{(\theta)}(x) \sum_{s=0}^{\rho_{2}} p_{\rho_{2}, s}^{(\theta)}(x) p_{m, \mu+s \rho_{1}}(t) .
$$

Lemma 5. For a fixed $x \in(0,1)$ and sufficiently large $m$, we have

(i) $\lambda_{m, \rho_{1}, \rho_{2}}^{(\theta)}(x, y)=\int_{0}^{y} \mathcal{N}_{m, \rho_{1}, \rho_{2}}^{(\theta)}(x, t) d t \leq \frac{\mathcal{W}_{\rho_{1}, \rho_{2}}^{(\theta)}}{(m+2)} \frac{x(1-x)}{(x-y)^{2}}, 0 \leq y<x ，$

(ii) $1-\lambda_{m, \rho_{1}, \rho_{2}}^{(\theta)}(x, z)=\int_{z}^{1} \mathcal{N}_{m, \rho_{1}, \rho_{2}}^{(\theta)}(x, t) d t \leq \frac{\mathcal{W}_{\rho_{1}, \rho_{2}}^{(\theta)}}{(m+2)} \frac{x(1-x)}{(z-x)^{2}}, x<z<1$,

where $\mathcal{W}_{\rho_{1}, \rho_{2}}^{(\theta)}$ is given in Lemma 3 .

Proof. (i) From Lemma 3, we get

$$
\begin{aligned}
\lambda_{m, \rho_{1}, \rho_{2}}^{(\theta)}(x, y) & =\int_{0}^{y} \mathcal{N}_{m, \rho_{1}, \rho_{2}}^{(\theta)}(x, t) d t \leq \int_{0}^{y}\left(\frac{x-t}{x-y}\right)^{2} \mathcal{N}_{m, \rho_{1}, \rho_{2}}^{(\theta)}(x, t) d t \\
& =U_{m, \rho_{1}, \rho_{2}}^{(\theta)}\left((t-x)^{2} ; x\right)(x-y)^{-2} \leq \frac{\mathcal{W}_{\rho_{1}, \rho_{2}}^{(\theta)}}{(m+2)} \frac{x(1-x)}{(x-y)^{2}}
\end{aligned}
$$

The (ii) can be proved in the same way hence the details are skipped. 
Theorem 8. Suppose that $\mathcal{S} \in D B V(j)$. Then for every $x \in(0,1)$ and sufficiently large $m$, we have

$$
\begin{aligned}
\left|U_{m, \rho_{1}, \rho_{2}}^{(\theta)}(\mathcal{S} ; x)-\mathcal{S}(x)\right| \leq & \frac{(1-2 x)}{(m+2)} \frac{\left|\mathcal{S}^{\prime}(x+)+\mathcal{S}^{\prime}(x-)\right|}{2}+\sqrt{\frac{\mathcal{W}_{\rho_{1}, \rho_{2}}^{(\theta)} x(1-x)}{(m+2)}} \frac{\left|\mathcal{S}^{\prime}(x+)-\mathcal{S}^{\prime}(x-)\right|}{2} \\
& +\frac{\mathcal{W}_{\rho_{1}, \rho_{2}}^{(\theta)}(1-x)}{(m+2)} \sum_{s=1}^{[\sqrt{m}]} \bigvee_{x-(x / s)}^{x}\left(\mathcal{S}_{x}^{\prime}\right)+\frac{x}{\sqrt{m}} \bigvee_{x-(x / \sqrt{m})}^{x}\left(\mathcal{S}_{x}^{\prime}\right) \\
& +\frac{\mathcal{W}_{\rho_{1}, \rho_{2}}^{(\theta)} x}{(m+2)} \sum_{s=1}^{[\sqrt{m}} \bigvee_{x}^{x+((1-x) / s)}\left(\mathcal{S}_{x}^{\prime}\right)+\frac{(1-x)}{\sqrt{m}} \bigvee_{x}^{x+((1-x) / \sqrt{m})}\left(\mathcal{S}_{x}^{\prime}\right),
\end{aligned}
$$

where $\bigvee_{c}^{d}\left(\mathcal{S}_{x}^{\prime}\right)$ denotes the total variation of $\mathcal{S}_{x}^{\prime}$ on $[c, d]$ and $\mathcal{S}_{x}^{\prime}$ is defined by

$$
\mathcal{S}_{x}^{\prime}(t)=\left\{\begin{array}{cc}
\mathcal{S}^{\prime}(t)-\mathcal{S}^{\prime}(x-), & 0 \leq t<x \\
0, & t=x \\
\mathcal{S}^{\prime}(t)-\mathcal{S}^{\prime}(x+) & x<t<1
\end{array}\right.
$$

Proof. This theorem can be proved in the same way as in ([4], Theorem 7). Hence, the proof of this theorem is skipped.

\section{Numerical Examples}

In the following examples, we demonstrate the theoretical results by graphs.

Example 1. Let $m=10, \rho_{1}=\rho_{2}=1$ and $\theta=0.5,0.6,0.7,0.8,1.0$. The convergence of the operators $U_{10,1,1}^{(0.5)}(\mathcal{S} ; x), U_{10,1,1}^{(0.6)}(\mathcal{S} ; x), U_{10,1,1}^{(0.7)}(\mathcal{S} ; x), U_{10,1,1}^{(0.8)}(\mathcal{S} ; x)$ and $U_{10,1,1}^{(1.0)}(\mathcal{S} ; x)$ to the function $\mathcal{S}(x)=x^{2} e^{\frac{x^{3}}{x+5}}$ is illustrated in Figure 1.

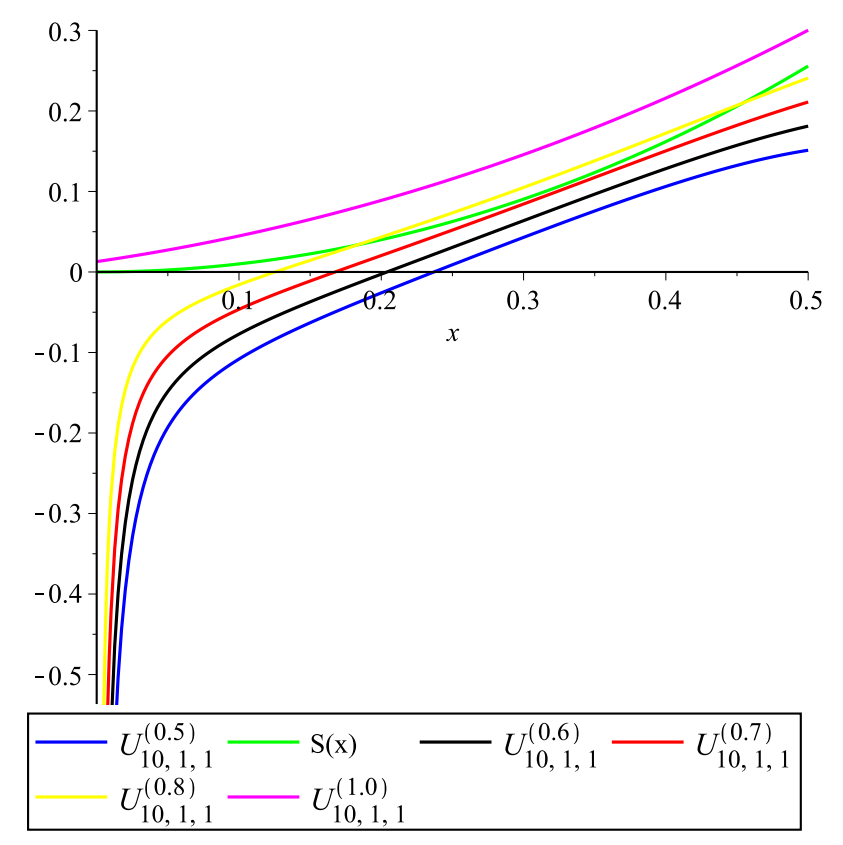

Figure 1. Approximation Process.

Example 2. Let $m=50, \rho_{1}=\rho_{2}=1$ and $\theta=0.5,0.6,0.7,0.8,1.0$. The convergence of the operators $U_{50,1,1}^{(0.5)}(\mathcal{S} ; x), U_{50,1,1}^{(0.6)}(\mathcal{S} ; x), U_{50,1,1}^{(0.7)}(\mathcal{S} ; x), U_{50,1,1}^{(0.8)}(\mathcal{S} ; x)$ and $U_{50,1,1}^{(1.0)}(\mathcal{S} ; x)$ to the function $\mathcal{S}(x)=x^{3} e^{\frac{x^{2}}{x+10}}$ is illustrated in Figure 2. 


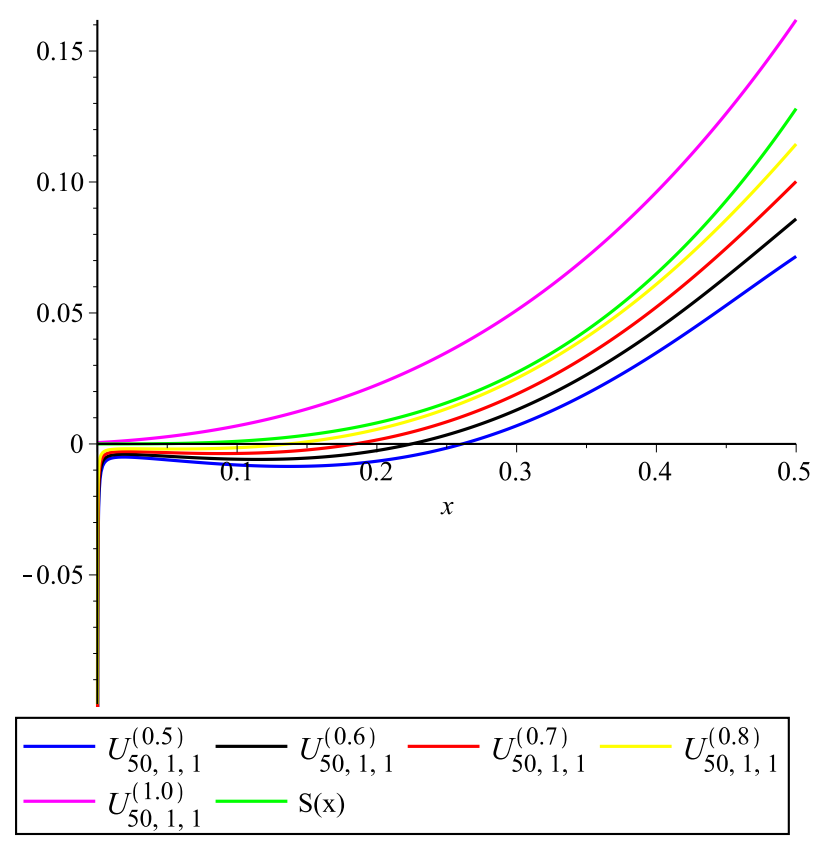

Figure 2. Approximation Process.

\section{Conclusions}

We have introduced generalized Bernstein-Durrmeyer type operators depending on non-negative integers. We developed many approximation properties such as local and global approximation, the rate of approximation for the Lipschitz type space, Voronovskaja type asymptotic formula and the rate of convergence of functions with derivatives of bounded variation. The constructed operators have better flexibility and rate of convergence which are depending on the selection of the $\rho_{1}, \rho_{2}$ and $\theta$. Graphical representations of our operators for different selections of $\rho_{1}, \rho_{2}$ and $\theta$ are also given.

Author Contributions: Project administration, M.M.; Writing—original draft, A.K.; Writing—review and editing, T.A. \& M.M. The authors contributed equally and significantly in writing this paper. All authors have read and agreed to the published version of the manuscript.

Funding: The third author has been partially supported within TUBITAK (The Scientific and Technological Research Council of Turkey) 1002-Project 119F191.

Conflicts of Interest: The authors declare no conflict of interest.

\section{References}

1. Weierstrass, K. Über die analytische Darstellbarkeit sogennanter willkürlicher Functionen einer reellen Veränderlichen. Sitzungsber. Akad. Berlin 1885, 633-639, 789-805.

2. Bernstein, S.N. Démonstation du théorème de Weierstrass fondée sur le calcul de probabilités. Commun. Soc. Math. Kharkow 1912, 13, 1-2.

3. Chen, X.; Tan, J.; Liu, Z.; Xie, J. Approximation of functions by a new family of generalized Bernstein operators. J. Math. Anal. Appl. 2017, 450, 244-261. [CrossRef]

4. Kajla, A.; Acar, T. Blending type approximation by generalized Bernstein-Durrmeyer type operators. Miskolc Math. Notes 2018, 19, 319-336. [CrossRef]

5. Acar, T.; Acu, A.M.; Manav, N. Approximation of functions by genuine Bernstein-Durrmeyer type operators. J. Math. Inequal. 2018, 12, 975-987. [CrossRef]

6. Abel, U.; Heilmann, M. The complete asymptotic expansion for Bernstein-Durrmeyer operators with Jacobi weights. Mediterr. J. Math. 2004, 1, 487-499. [CrossRef]

7. Cárdenas-Morales, D.; Gupta, V. Two families of Bernstein-Durrmeyer type operators. Appl. Math. Comput. 2014, 248, 342-353. [CrossRef] 
8. Abel, U.; Ivan, M.; Paltanea, R. The Durrmeyer variant of an operator defined by D.D. Stancu. Appl. Math. Comput. 2015, 259, 116-123.

9. Agrawal, P.N.; Goyal, M.; Kajla, A. q-Bernstein-Schurer-Kantorovich type operators. Boll. Unione Mat. Ital. 2015, 8, 169-180. [CrossRef]

10. Ansari, K.J.; Mursaleen, M.; Rahman, S. Approximation by Jakimovski-Leviatan operators of Durrmeyer type involving multiple Appell polynomials. Rev. R. Acad. Cienc. Exactas Fis. Nat. Ser. A Mat. RACSAM 2019, 113, 1007-1024. [CrossRef]

11. Acar, T.; Aral, A.; Rasa, I. Positive linear operators preserving $\tau$ and $\tau^{2}$. Constr. Math. Anal. 2019, 2, 98-102.

12. Mursaleen, M.; Rahman, S.; Ansari, K.J. Approximation by Jakimovski-Leviatan-Stancu-Durrmeyer type operators. Filomat 2019, 33, 1517-1530. [CrossRef]

13. Acu, A.M.; Kajla, A. Blending Type Approximation by Generalized Bernstein Operators; 2020, submitted for publication.

14. Korovkin, P.P. On convergence of linear positive operators in the space of continuous functions. Dokl. Akad. Nouk. SSR 1959, 90, 961-964. (In Russian)

15. Acar, T.; Aral, A. The new forms of Voronovskaya's theorem in weighted spaces. Positivity 2016, $20,25-40$. [CrossRef]

16. Acar, T. Quantitative q-Voronovskaya and q-Grüss-Voronovskaya-type results for q-Szasz operators. Georgian Math. J. 2016, 23, 459-468. [CrossRef]

17. Erencin, A.; Rasa, I. Voronovskaya type theorems in weighted spaces. Numer. Funct. Anal. Optim. 2016, 37, 1517-1528. [CrossRef]

18. Ulusay, G.; Acar, T. q-Voronovskaya type theorems for q-Baskakov operators. Math. Method Appl. Sci. 2016, 39, 3391-3401. [CrossRef]

19. Özarslan, M.A.; Aktuğlu, H. Local approximation for certain King type operators. Filomat 2013, $27,173-181$. [CrossRef]

20. Devore, R.A.; Lorentz, G.G. Constructive Approximation, Grundlehren der Mathematischen Wissenschaften; Springer: Berlin, Germany, 1993; Volume 303.

21. Ditzian, Z.; Totik, V. Moduli of Smoothness; Springer: New York, NY, USA, 1987.

(C) 2020 by the authors. Licensee MDPI, Basel, Switzerland. This article is an open access article distributed under the terms and conditions of the Creative Commons Attribution (CC BY) license (http://creativecommons.org/licenses/by/4.0/). 